Intelligent documentation as a catalyst for developing cooperative knowledge-based systems

Franz Schmalhofer, Thomas Reinartz, Bidjan Tschaitschian

June 1992

Deutsches Forschungszentrum für Künstliche Intelligenz $\mathrm{GmbH}$

Postfach 2080

D-6750 Kaiserslautern, FRG

Tel.: (+49 631) 205-3211/13

Fax: (+49 631) 205-3210
Stuhlsatzenhausweg 3

D-6600 Saarbrücken 11, FRG

Tel.: (+49 681) 302-5252

Fax: (+49 681) 302-5341 


\section{Deutsches Forschungszentrum für Künstliche Intelligenz}

The German Research Center for Artificial Intelligence (Deutsches Forschungszentrum für Künstliche Intelligenz, DFKI) with sites in Kaiserslautern and Saarbrücken is a non-profit organization which was founded in 1988 by the shareholder companies Daimler-Benz, IBM, Insiders, Fraunhofer Gesellschaft, GMD, Krupp-Atlas, Digital-Kienzle, Philips, Sema Group Systems, Siemens and Siemens-Nixdorf. Research projects conducted at the DFKI are funded by the German Ministry for Research and Technology, by the shareholder companies, or by other industrial contracts.

The DFKI conducts application-oriented basic research in the field of artificial intelligence and other related subfields of computer science. The overall goal is to construct systems with technical knowledge and common sense which - by using Al methods - implement a problem solution for a selected application area. Currently, there are the following research areas at the DFKI:

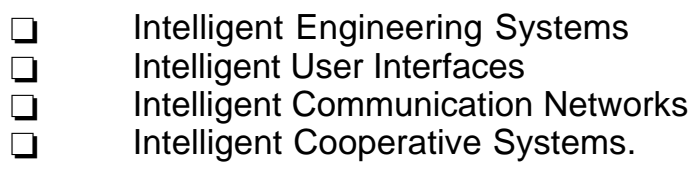

The DFKI strives at making its research results available to the scientific community. There exist many contacts to domestic and foreign research institutions, both in academy and industry. The DFKI hosts technology transfer workshops for shareholders and other interested groups in order to inform about the current state of research.

From its beginning, the DFKI has provided an attractive working environment for Al researchers from Germany and from all over the world. The goal is to have a staff of about 100 researchers at the end of the building-up phase.

Prof. Dr. Gerhard Barth

Director 
Intelligent documentation as a catalyst for developing cooperative knowledge-based systems

Franz Schmalhofer, Thomas Reinartz, Bidjan Tschaitschian

DFKI-RR-92-26 
This paper has been published in the Conference Proceedings of the 6th European Knowledge Acquisition Workshop: Th. Wetter, K.-D. Althoff, J. Boose, B.R. Gaines, M. Linster, F. Schmalhofer (Eds.), "Current Developments in Knowledge Acquisition - EKAW'92", Springer-Verlag, Berlin 1992.

This work has been supported by a grant from The Federal Ministry for Research and Technology (FKZ ITW-8902 C4).

(C) Deutsches Forschungszentrum für Künstliche Intelligenz 1992

This work may not be copied or reproduced in whole or in part for any commercial purpose. Permission to copy in whole or in part without payment of fee is granted for nonprofit educational and research purposes provided that all such whole or partial copies include the following: a notice that such copying is by permission of Deutsches Forschungszentrum für Künstliche Intelligenz, Kaiserslautern, Federal Republic of Germany; an acknowledgement of the authors and individual contributors to the work; all applicable portions of this copyright notice. Copying, reproducing, or republishing for any other purpose shall require a licence with payment of fee to Deutsches Forschungszentrum für Künstliche Intelligenz. 


\section{Contents}

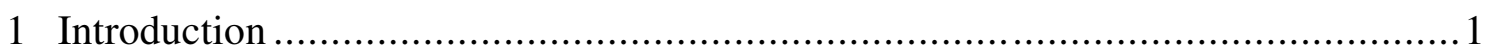

2 The Real-World Domain of Mechanical Engineering ....................................2

2.1 The Manufactoring of Rotational Parts ..............................................2

2.2 Characterization of the Relevant Information Sources .......................... 4

3 Integrated Knowledge Acquisition Method ............................................ 4

3.1 Basic Requirements ....................................................... 5

3.2 Aquisition Method .................................................................6 6

3.3 The Acquisition of Problem Classes and Operator Classes with CECoS ....8

3.4 Case Oriented Knowledge Acquisition with SP-GEN ......................... 12

4 Domain- and Task-Specific Hypertext as Intelligent Documentation...................... 13

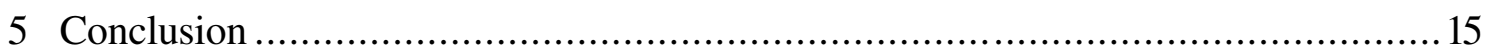

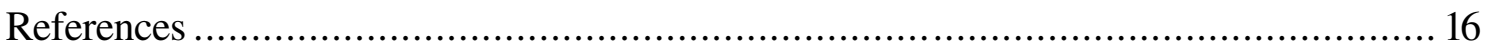




\title{
Intelligent Documentation as a Catalyst for Developing Cooperative Knowledge-Based Systems
}

\author{
Franz Schmalhofer, Thomas Reinartz, and Bidjan Tschaitschian \\ German Research Center for Artificial Intelligence \\ University Building 57 \\ Erwin-Schrödinger Str. \\ W-6750 Kaiserslautern \\ Germany \\ e-mail: schmalho@informatik.uni-kl.de
}

\begin{abstract}
In the long run, the development of cooperative knowledge-based systems for complex real world domains such as production planning in mechanical engineering should yield significant economic returns. However, large investments have already been made into the conventional technology. Intelligent documentation, which abstracts the current practice of the industry, is suggested as a stepping stone for developing such knowledge-based systems. A set of coordinated knowledge acquisition tools has been developed by which intelligent documents are constructed as an intermediate product, which by itself is already useful. Within the frame of the conventional technology, the task- and domain specific hypertext structures allow the reuse of production plans while simultaneously starting the development process for knowledge based systems.
\end{abstract}

\section{Introduction}

While the specific needs of human problem solvers have often been ignored in expert systems, the pivotal importance of system-user interactions is now well recognized. The more recently developed cooperative knowledge-based systems indeed support the users in their problem solving and learning activities (Fischer, Lemke, Mastaglio \& Morch, 1990; Reiser et al. 1988).

The application of such concepts to complex real world problems would yield significant economic benefits in the long run. With these knowledge-based systems a user could solve complex problems faster and more reliably. In addition, the expertise of the domain would be better documented, which would facilitate its communication and also allows the reuse of old problem solutions in related contexts.

Nevertheless, the potential sponsors and customers of cooperative knowledge-based systems hesitate to introduce this innovation on a large scale to their companies. Since the conventional procedures, which are currently in practice are perceived as quite adequate, hardly anybody feels a need for change. Practitioners may even fear the uncertainties, which could arise when knowledge-based systems are introduced into their field. When innovation is introduced the established competence of a company could even be lost. Maturing innovative approaches so that they work well in practice requires long development periods which are very expensive and disruptive to the current technology. Potential customers may therefore doubt that an innovation can be successfully applied to their domain. There is obviously a high barrier for introducing knowledge-based systems into the industry.

This barrier is particularly severe for complex real world domains, where sophisticated competence has been accumulated over long time periods. For example the competence in the field of mechanical engineering is extremely elaborate and detailed and has matured over at least hundred years (Spur, 1979). 
Over the last few years our knowledge acquisition group has developed an integrated knowledge acquisition method. The current paper describes how an intelligent documentation in form of a domain-specific hypertext structure is formed with this method as an intermediate knowledge representation. Since such an intelligent documentation will yield direct benefits in its own right, it will lower the barrier for introducing knowledge-based systems. First we describe the complex real world domain of mechanical engineering, in order to show the necessity of an intelligent documentation. Thereafter the integrated knowledge acquisition method with its three coordinated tools is explained, and the resulting domain- and task-specific hypertext as the intelligent documentation is presented.

\section{The Real-World Domain of Mechanical Engineering}

In the field of mechanical engineering numerous descriptions exist as written documents and through the explanations of various domain experts. Such descriptions are usually uncommittal: imprecisely stated, at different levels of generality, overall incomplete and at times even contradictory. In other words, the knowledge of the field is quite badly documented. This knowledge is nevertheless needed for solving specific problems such as for example the manufacturing of rotational parts. Because of the quantity and variety of knowledge which enters into solving such problems, the planning of the manufacturing process is itself complex.

\subsection{The Manufacturing of Rotational Parts}

The technique for manufacturing a rotational part is best understood by a comparison to pottery. The manufacturing processes are similar to making a pot in the following way: One puts or attaches a piece of clay to a potter's wheel and shapes the clay to a specific form, only by removing some parts of the clay while the potter's wheel is turned. Contrary to the soft clay, which also allows a potter to push some material to a neighboring position, a rota-tional part or workpiece (metals) is shaped, solely by removing materials with a hard cutting tool.

Figure 1 shows a graphical representation of a (partial) workplan for a rotational part. The geometric form of the mold and the target workpiece are overlayed and shown at the top part of the figure (in the middle). The chucking fixture (seen as the black area on the left and the black triangle on the right side) is rotated with the attached mold (a $500 \mathrm{~mm}$ long cylinder indicated by the shaded area) with the longitudinal axis of the cylinder as the rotation center. The sequence of cuts are indicated by the numbers 1 to 7 . For each cut the cutting tool, the cutting parameters, and the cutting path are also shown in the figure. For example, the cutting tool number 1 has the specification "CSSNL 3232 C15 SNGN151016 TO 3030". It is applied to remove a part of the upper layer of the cylinder with a rotation speed of $\mathrm{v}_{\mathrm{c}}=450 \mathrm{~m} / \mathrm{min}$, a feed of $\mathrm{f}=0,45 \mathrm{~mm} / \mathrm{U}$ and a cutting depth of $\mathrm{ap}=5 \mathrm{~mm}$. A complete description of the real world operations would also include further technological data of the workpiece (surface roughness, material, etc.) and precise workshop data (CNC machines with their rotation power and number of tools and revolvers, etc.).

This plan is relatively simple in comparison to the planning processes by which it was generated. Human experts require several hours to produce the first complete version of the plan. A total of two days is spent before it is successfully tested and a qualitatively good plan is obtained. The following facts of the application domain may explain why these planning processes are so time consuming.

About $1.8 * 10^{7}$ toolholders and $1.5 * 10^{8}$ tool inserts can be specified within the ISO norm 5608 and the ISO norm 1832 . However, only $3.5 * 1012$ cutting tools are potentially usable. The very large number $(2.6965 * 1015)$ of meaningless specifications (i.e. specifications for 


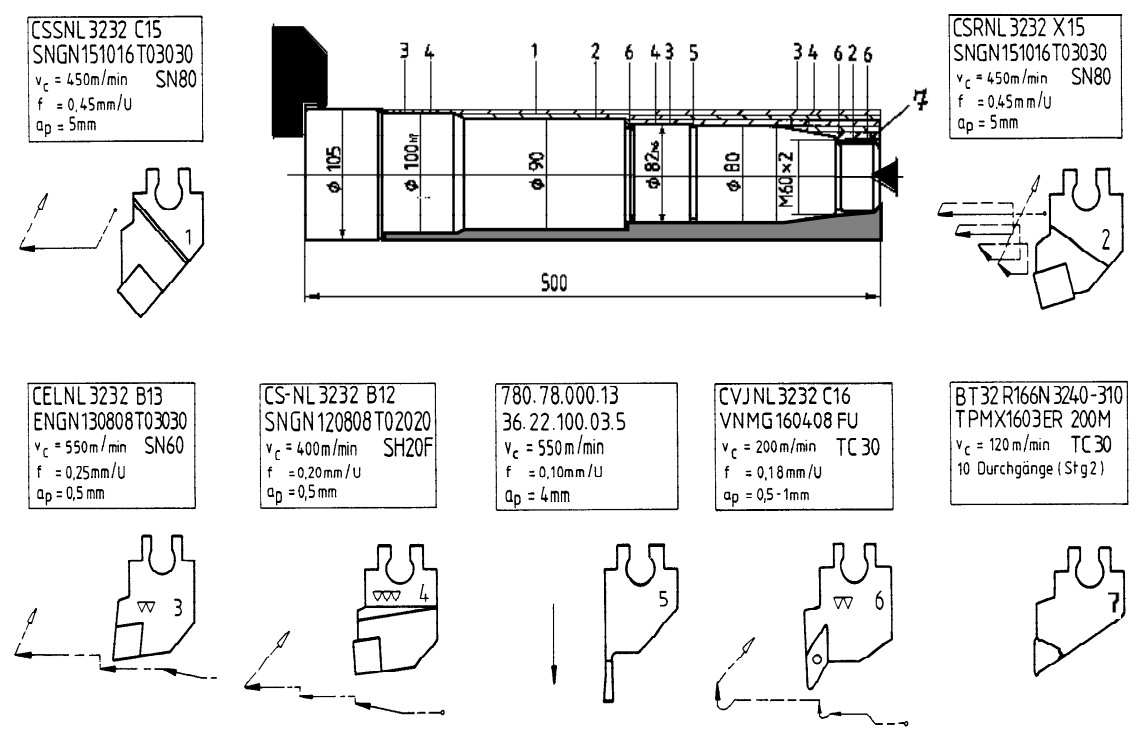

Figure 1: Graphical representation of a typical workplan for a rotational part (From "Examples for Application" (pp. 25-27), Plochingen Neckar, Germany: Feldmühle AG. Copyright 1984 by Feldmühle AG. Reprinted by permission.)

which no cutting tool can be physically assembled) shows the inadequacy of the ISO notations. From the potentially useful cutting tools 40000 tools are commercially available and a medium sized company will typically apply 5000 tools in their production. Some highly specialized cutting tools may in addition specifically be constructed for the manufacturing of some workpiece. A workplan typically uses between 6 and 16 tools. Since a number of parameters have to be determined from a continuum (chucking force, cutting speed, feed and path, etc.) the number of possible alternatives can be considered arbitrarily large. Depending on the various cutting parameters (see Figure 1) each of the tools can be used in different ways to produce different effects. Although they provide a structure into which the tools can be sorted, the existing ISO norms are not sufficiently tailored to the needs of the planning process.

The production plan must also fit the specific CNC machine which is used for manufacturing the workpiece. For each company the CNC machines are individually configured from a set of different components. The configuration of a machine depends on the spectrum of workpieces and the lot size which the company expects to produce. Therefore rarely two lathe machines of a company are completely identical.

A company has a typical history and profile with respect to the workpieces it usually produces. The typical manufacturing cases of a company can therefore be found in firm records so that they can be reused when a similar workpiece needs to be produced.

There are a number of interdependencies between the tools, the CNC machines and the workpieces to be produced. CNC machines must have a large enough revolver to keep all the necessary tools. In addition, the $\mathrm{CNC}$ machine must have enough power to achieve the required cutting speed and force for the operations specified in the plan.

Since the quality of the resulting plan is very high whereas the planning processes themselves are not completely knowledge-based in the sense that the plans have to be developed to some degree by trial and error in the real world, human experts often obtain a new plan by modifying previous planning products to the new problems. The information and the knowledge which is needed for production planning is distributed among at least three different sources. 


\subsection{Characterization of the Relevant Information Sources}

Human Experts. Human experts are known to form and utilize concept hierarchies (Chase \& Ericcson, 1982; Chi, Feltovich \& Glaser, 1981) in such a way that the most useful distinctions are made for their domain of expertise (de Groot, 1966). Within this concept hierarchy there exist so called basic level categories (Rosch, 1978; Hoffmann \& Zießler, 1983). These categories tend to be formed so that the associated task related operations can be successfully applied to the members of each category (Chi, Feltovich \& Glaser, 1981). An expert's concept hierarchy should thus have uniform solution methods stored with each concept (Anderson 1990, Chapter 3 ). Usually, these solution methods consist of compiled knowledge and cannot be directly expressed verbally. An expert's judgements about prob-lem similarities may, however, be used to infer the underlying hierarchical organization.

Written Documents. In mechanical engineering written documents exist in the form of text books for engineering students, practical guidelines for the various applications in the domain and catalogues from tool manufacturers. The text books present the relevant knowledge from geometry and physics in a task independent way such as the calculation of angles, the decomposition of forces and classifications of materials on the basis of physical and chemical properties. There are also descriptions of application specific models and summaries of experiments about the applicability of different types of tools. In the catalogues of the tool manufacturers, the more specific experimental results about the offered tools are published. The practical guidelines describe particular problems and present relevant criteria which should be considered when tackling these problems.

Solutions of Previous Manufacturing Problems. The solutions of previous manufacturing problems (i.e. manufacturing plans) which have been proven successful by numerous applications in the real world can be found in databases (or filing cabinets) of the different manufacturers. A set of qualitatively good cases which is representative of the de-sired competence of the future knowledge based system can provide important information.

The large amount of different types of information, which are used according to some conventional procedures, emerged over a long development period. The large number of interdependencies among the components of the planning procedure and the different types of information also make it difficult to introduce a differently structured innovative procedure, without a long period of preparation. Because of the forbidding high investment cost, it is almost impossible to successfully introduce knowledge-based systems into such complex real world domains by a single software project.

In order to overcome this barrier, one would therefore need to make some preparatory steps, which are beneficial in their own right. We thus suggest to define a milestone for introducing knowledge-based systems, which, however, can be seen as a goal of its own. An intelligent documentation of the actual procedures, which is organized according to the structure of a possibly future knowledge-based system is such an intermediate step. The following section describes how an integrated knowledge acquisition method can be used to develop an intelligent documentation of production planning, from which a knowledge-based system can be subsequently constructed.

\section{Integrated Knowledge Acquisition Method}

When knowledge is independently acquired from different, but equally respectable sources (e.g. different experts, different information sources) or with different and equally profitable tools, knowledge may be integratively analyzed. Since several relevant information sources have been identified, an integrated knowledge acquisition method has been developed, that uses several tools to accomplish intelligent documentation and an efficient utilization of these sources, as well as an early verification of the elicited knowledge. 
The purpose of intelligent documentation as well as documentation in general is to support users in their problem solving activities. In order to support the human problem solving strategies for production planning, an intelligent documentation of the relevant information sources should be oriented towards the human problem solving activities.

In the following, some important issues from investigations of expert's problem solving behavior are presented, resulting in a model of expertise for production planning. Then the requirements for an integrative approach are addressed, followed by the introduction of the integrated knowledge acquisition method. Finally, a brief description of their tools is given.

\subsection{Basic Requirements}

Supposedly an expert's memories include a hierarchy of workpiece classes. Results from cognition (Chi, Feltovich \& Glaser, 1981; Egan \& Schwartz, 1979; de Groot, 1966; Chase \& Ericcson, 1982) provide substantial evidence that these classes have been formed according to skeletal production plans.

In an empirical investigation (Thoben, Schmalhofer \& Reinartz 1991), and a related thinkaloud study (Schmidt, Legleitner \& Schmalhofer, 1990), the expert's problem solving behavior for finding a production plan was investigated in detail. The results showed that nearly all production planning can be seen as modification planning, i.e. a more or less abstract skeletal plan (Friedland \& Iwasaki, 1985; Chien, 1989) is retrieved and subsequently refined to a specific workplan. When the skeletal plan is quite general, skeletal plan refinement, which relies on generative planning processes, plays the major role. When the skeletal plan is so specific that it is very close to a detailed production plan, the memory retrieval accomplishes the planning goal (replication planning). Replication planning is thus a special case of modification planning (Hammond, 1989), where a complete plan is already available. Between these two extremes the various forms of modification planning occur, where the planning effort is differently distributed between the retrieval of the skeletal plan and its refinement.

Model of Expertise. From these investigations a model of expertise (Breuker \& Wielinga, 1989) has been developed (see Figure 4, left side): The problem of production planning consists of finding an adequate production plan for a given workpiece which is to be manufactured in some given factory. The description of the workpiece is presented by the workpiece model and the description of the factory (i.e. the available machines and tools) by the factory model. From these concrete data an abstract feature description of the workpiece and an abstract context specification are obtained through the application of abstraction or classification rules. To these abstract workpiece and context descriptions a skeletal plan can be associated which may be seen as an abstraction of a concrete production plan. The skeletal plan is then refined with the help of the workpiece and the factory models so that an executable production plan is obtained.

In order to develop a documentation which fits the described human problem solving strategies, the different information sources should be documented with respect to this model, i.e. elicited knowledge units have to be categorized according to the knowledge sources and meta-classes in the model of expertise.

With the previously developed integrated knowledge acquisition method (Schmalhofer, Kühn \& Schmidt, 1991) the relevance and sufficiency of the elicited knowledge, and problems with redundancies and contradictions can be assessed. Consequently, a respective intelligent documentation will also provide an assessment of the relevance and sufficiency of the information and the elimination of redundancies and contradictions.

Relevance and Sufficiency Assessment. The relevance and the sufficiency of general knowledge units can be determined by having an expert explain prototypical target tasks (cases) with the general knowledge units. These units (e.g. general statements from a text or general experiences from human experts) are elicited, because of their great generality and high reliability. High quality task solutions are selected, so that the specific set of examples forms a 
base for all problems which the expert system is supposed to solve. Because the set of examples defines the particular area of competence of the knowledge-based system, they can be used as a guide for selecting those general knowledge units which constitute the relevant knowledge. By having the expert instantiate the general knowledge with respect to the specific cases and by performing constructive explanations, a bridge is built between the general knowledge units and the specific cases. Thereby a knowledge integration can be performed between a case base and a text, or a case base and general expert experiences. Relevance and sufficiency are consequently assessed with respect to the selected cases.

Elimination of Redundancies and Contradictions. In order to eliminate major redundancies and overt contradictions the various knowledge units must be compared in a systematic way. The model of expertise together with the domain oriented decomposition into subtasks provides a useful categorization for the elicited knowledge units. By comparing the knowledge units of a category with all other knowledge units of the category the most critical redundancies and contradictions are eliminated.

Early Knowledge Verification. Through the application of these principles an early knowledge verification is performed. With informally presented knowledge (e.g. natural language sentences and rules), which is often more comprehensible to a human expert (Schmidt \& Wetter, 1989) relevance, sufficiency, redundancies and contradictions can be identified relatively soon. Formal considerations (i.e. completeness and consistency of the knowledge base) are thereby already treated with informally denoted knowledge. Performing such formal considerations at an informal level is termed early knowledge verification.

\subsection{Acquisition Method}

These requirements are met by the integrated knowledge acquisition method (Schmalhofer, Kühn \& Schmidt, 1991), which has been developed for constructing cooperative knowledgebased systems. This general method can be applied for constructing an intelligent documentation. We propose to combine knowledge units, elicited from several information sources, with different acquisition tools and on various levels of abstraction, with respect to the model of expertise and the elicitation history. Thus we get means to provide high-level explanation facilities (Yetim, 1991) and to store suitable information about interdependencies of the knowledge units.

In the following we describe two coordinated knowledge acquisition tools, which were designed to construct an intelligent documentation on route to a formal knowledge base: The case experience combination system or CECoS (Bergmann \& Schmalhofer, 1991) and the case oriented knowledge acquisition method from texts or COKAM+ (Schmidt \& Schmalhofer, 1990). After the documented information has been formalized a skeletal plan generation procedure SP-GEN (Schmalhofer, Bergmann, Kühn \& Schmidt, 1991) can be applied to construct skeletal plans for the different problem classes.

Figure 2 shows how CECoS and COKAM+ are used to construct an intelligent documentation of human problem solving strategies and related information for production planning on route to the development of a knowledge-based system. A hierarchy of problem classes from the set of selected cases and a hierarchy of operator classes from the set of operations, which occur in the plan of the selected cases, are delineated by CECoS.

With COKAM+ the set of operations and the operator classes which have so far only been delineated by their extensions are now specified in terms of preconditions and consequences, which have to be formalized to STRIPS-like operator definitions. The preconditions and consequences at the various levels of generality are related by common sense and engineering knowledge. Both types of knowledge are interactively extracted from written documents. 


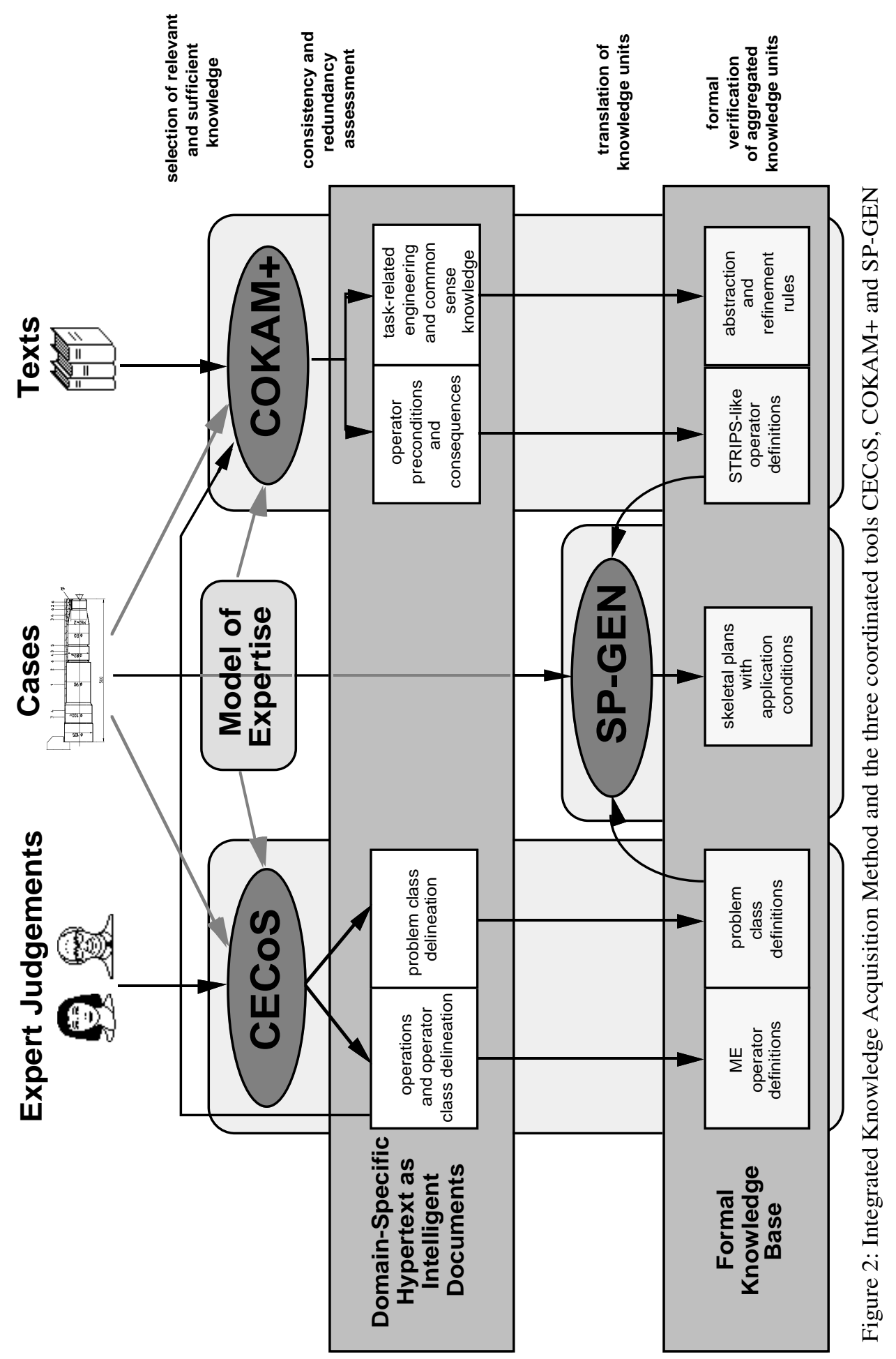


The knowledge acquisition tools COKAM+ and CECoS are applied to the same set of cases so that the knowledge acquired with these tools will complement one another. The domain and common sense knowledge is supplied by COKAM+. The definition of operator classes and the definition of production classes are obtained through CECoS. The white boxes of Figure 2 show the global structure of the intelligent documentation and the grey boxes below show the corresponding entities in the knowledge-based system.

\subsection{The Acquisition of Problem Classes and Operator Classes with CECoS}

With the interactive tool CECoS hierarchically structured problem and operator classes are obtained which closely correspond to the hierarchical organization in the expert's memory.

Similarity Judgements. In the first step of the CECoS procedure a human expert judges the similarity of pairwise presented problems or operators on a discrete scale between 1 (very different) and 7 (very similar). Since a complete comparison yields $n *(n-1) / 2$ judgements for $n$ items, it may be useful to group items in classes with respect to global and obvious similarities as a primary step, and to take these classes as the items of the initial paired comparison. Later on CECoS may be applied to each class separately and the resulting tree structures can be joined (see Schmalhofer \& Thoben, in press). Thus the complexity is now basically linear instead of a polynomial increase with the number of items (cases or operators) being considered.

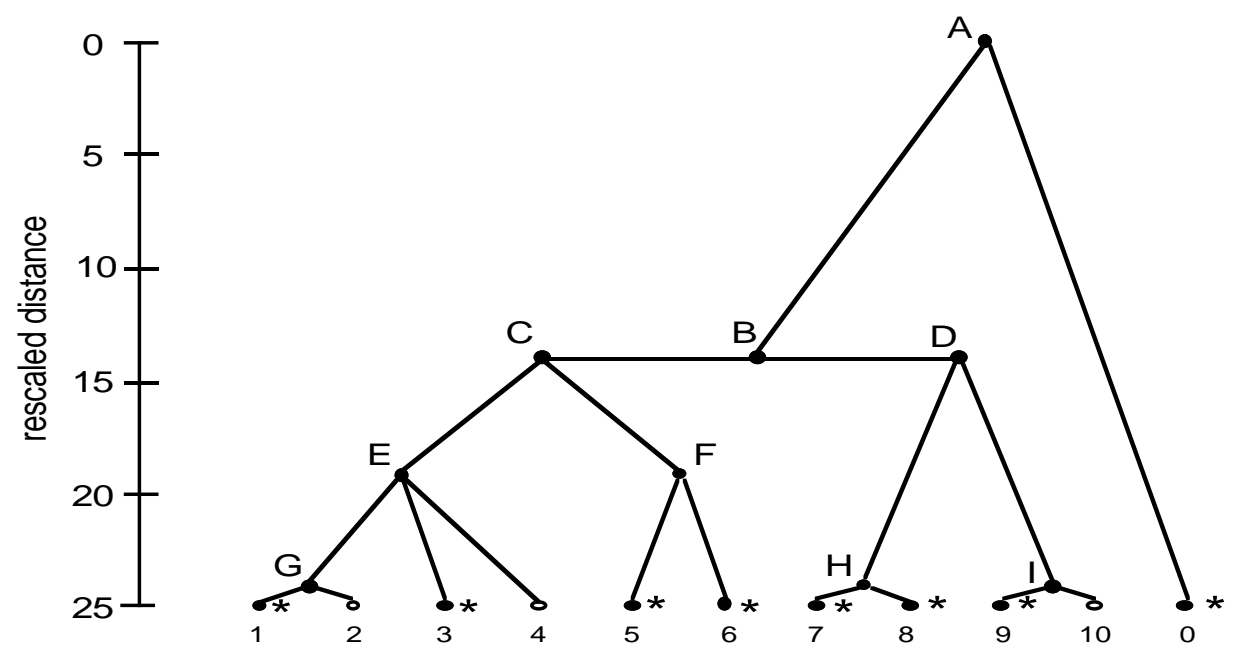

Figure 3: Resulting hierarchy of problem classes for rotational parts in mechanical engineering from an example application of CECoS

Hierarchical Cluster Analysis. A hierarchical cluster analysis is separately applied to each of the resulting similarity matrix. The outcome is a hierarchy of problem or operator classes which are extensionally defined by their reference to the items, for which similarity judgements were given in the first step. Each class subsumes its subordinate classes and instances, i.e. every item, which belongs to a certain class within the hierarchy, is also a member of all classes from the root to the specific class. Figure 3 shows such a hierarchy of problem classes for rotational parts, which was received by an example application to CECoS on 60 different cases, which varied in five geometries, four workpiece materials, and three turning machines. Each terminal problem class 0 to 10 consists of several single cases, which have been grouped in a primary step. The CECoS procedure has also been applied to the classes 5 and 6 (see Reinartz, 1991). The vertical distance between two classes within the hierarchy shows the grade of similarity. Thus the classes 1 and 2 are more similar than the classes 3 and 4. Classes marked by an asterisk include some cases with corresponding skeletal plans acquired in a related study (Thoben, Schmalhofer \& Reinartz, 1991; Schmalhofer \& Thoben, in press). 


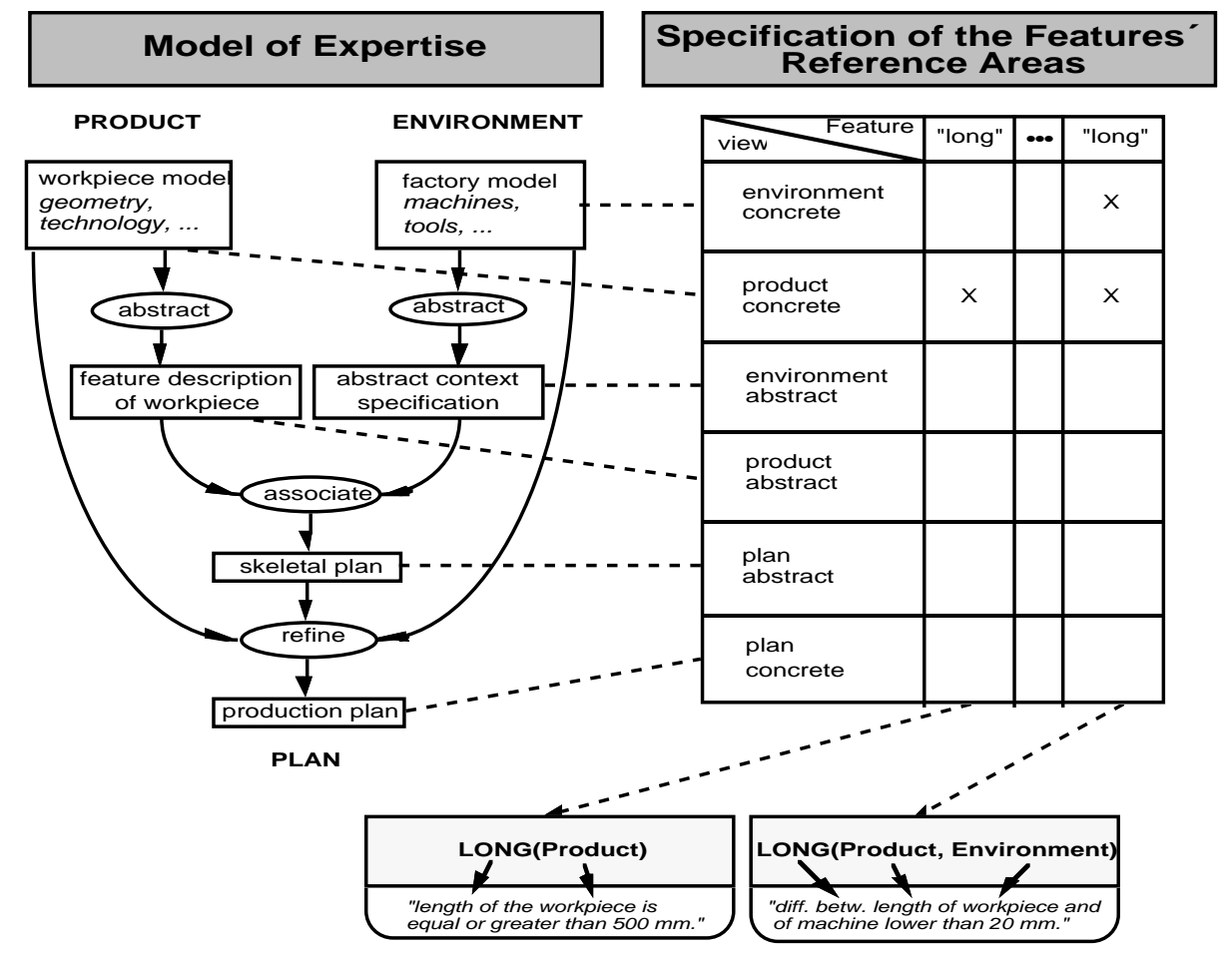

Figure 4: How the model of expertise yields different views and guides the intensional definitions of problem classes in CECoS

Acquisition of Features. After the extensional definition of the problem classes the expert is asked to define each class intensionally by naming several features. CECoS presents a certain class and the expert can type some features, but has to take into account that every named feature has to fit all subordinate problem classes, since the subsumption principle holds. For example "long workpiece" is a quite general feature, which belongs to class B (and hence to all classes in the subtree of class B), whereas "threat M60x2" is more precise and describes a terminal class in the (not shown) subtree of class 6. The operator classes, on the other hand, are intensionally defined within the tool COKAM+ (see section 3.4).

Views. Each feature can be seen as a single knowledge unit, which is used to explain a certain problem class. The next step on route to develop a formal knowledge-base is to structure these units according to the model of expertise. For large and complex real world domains such as mechanical engineering it is necessary to divide the knowledge base into several specific reference areas. This strategy can be compared with modularization for large programming tasks in Software Engineering (Wachsmuth \& Meyer-Fujara, 1990). The main reason for modularization is to reduce the complexity of the resulting system. A structured knowledge base may also easily be developed and changed, since each 'module' can be treated more or less independently. Thus the following steps in CECoS are less complex, if the knowledge base has been appropriately partitioned before.

A model of expertise (Kühn \& Schmalhofer, 1992) whose general structure is shown in Figure 4 guides our modularization of the knowledge base. Since each reference area can be interpreted as one special view of the knowledge base, we call our 'knowledge modules' views. Each rectangle of the model of expertise defines one single view. Thus we distinguish product, environment and plan view, and concrete and abstract views, so that all in all six single views exist. The expert has to assign every feature to one of these views by marking the corresponding field in a table (see Figure 4). For example the feature "long" with its absolute meaning is assigned to the single view 'concrete product view'. Since we do not expect, that every feature can be assigned clearly to one single view, because the different reference areas are not absolutely inde- 
pendent, we also allow the assignment of a feature to two or more views. Such assignments are called combined view assignments. For example the feature "long" in a relative sense (i.e. length as a difference between workpiece and turning machine) is such a combined view feature, because it relates 'concrete product view' and 'concrete environment view' to one another.

Consistency and Redundancy Assessment. After the features have been assigned to the various single and combined views a consistency and redundancy assessment is performed (Tschaitschian, 1991). This assessment is first performed separately for each view. After this local consistency and redundancy assessment is established, the respective checks can be made at a more global level. These consistency and redundancy checks are less complex through the modularization of the knowledge base, since not every possible interaction between two arbitrary features has to be considered, but only the designated ones.

Formalization. Within the last two steps the so far elicited knowledge base is formalized. First, in a semiformalization, the expert has to define his features in terms of more simple expressions. Each feature is presented with its views and the expert has to fill in his explanations in a template (see lower part of Figure 4). He is only allowed to use terms of the respective views, since the feature belongs to this part of the knowledge base. These explanations are then used to give a formal definition of the features, and the problem classes are consequently defined by the conjunction of the respective feature formalizations. The formalization is also structured by the different views, so that this task is similarly modularized to the consistency and redundancy assessment.

The choice of a sufficient knowledge representation language is important and has to suffer the requirements given by the procedure. A first attempt has been made by using a KL-ONE like representation system, but unfortunately it failed, since it was too inefficient, and not powerful enough to define the features in a sufficient manner (see Reinartz, 1991).

Because the class definitions are based on expert judgements, the classes should be defined at the right level of generality (Rosch, 1978): They should be general enough so that a large number of specific problems fall into the different classes and they should be specific enough to provide operational knowledge for production planning.

A detailed description of the example application to 60 cases named above and the formalization approaches in a KL-ONE like knowledge representation language are given in (Reinartz, 1991) and (Schmalhofer \& Thoben, in press).

\subsection{Case Oriented Knowledge Acquisition with COKAM+}

With the tool COKAM+ (see Schmidt, 1992) information about the complex machining operations is interactively extracted from a text and subsequently enhanced by the expert's elaborations. The extracted information is then mapped to a model of mechanical engineering actions (domain model) and in addition categorized according to the views which are derived from the model of expertise. The so collected knowledge thus provides an explanation of each step in the production plan and specifies the conditions which are required for its application and the resulting consequences.

Figure 5 shows an example from Schmidt (1992) which demonstrates how an operation is explained in terms of its preconditions and consequences. The particular operation (denotation of operation), that is explained here, is the first cut of the workplan that was shown in Figure 1. As can be seen from the first precondition node ("cutting speeds"), one of the general preconditions of this operation requires $\mathrm{SN}$-ceramics to be used with a cutting speed lower than $800 \mathrm{~m} / \mathrm{min}$. The nodes of the task-related engineering and common sense knowledge, that are linked to the first precondition node explain that the specific materials (SN80 and GGG90) fall into the required categories of SN ceramics and cast iron. The consequence nodes inform 


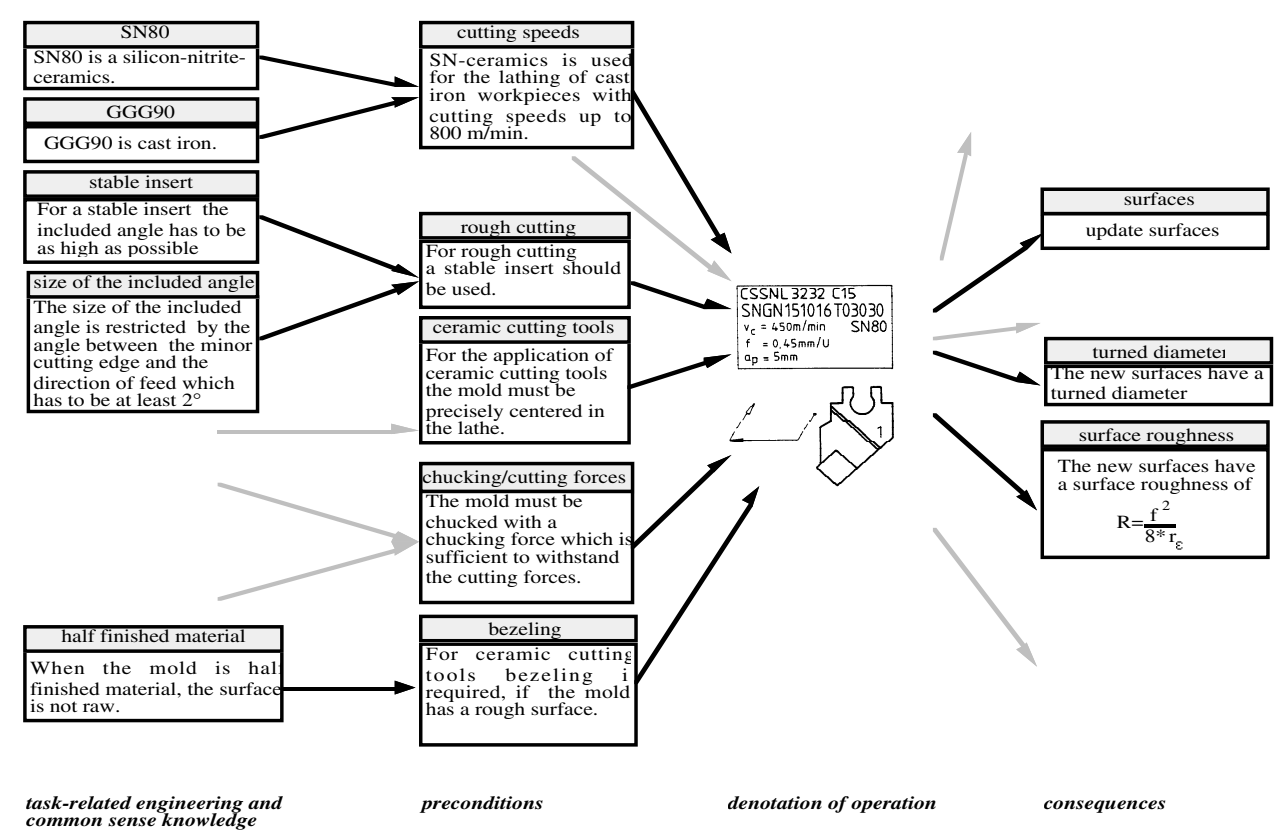

Figure 5: Documentation of the preconditions and consequences of operators with COKAM+ (after Schmidt, 1992)

how for example the surface roughness resulting from the operation is determined. From such precondition and consequence descriptions STRIPS-like ADD and DELETE lists can later on be derived for the formal knowledge base.

\subsection{Skeletal Plan Generation with SP-GEN}

The skeletal plan generation procedure (SP-GEN) (Schmalhofer, Bergmann, Kühn \& Schmidt, 1991) is based on explanation-based generalization as described by (Mitchell, Keller \& KedarCabelli, 1986). The domain and common sense knowledge acquired with COKAM+ is thereby used as the domain theory and the hierarchy of manufacturing classes is employed to specify operationality criteria. Depending upon the selected manufacturing class and the respective operationality criteria, a more or less general skeletal plan will be obtained from a given case. The problem classes elicited by CECoS are defined so that a useful skeletal plan will exist for each problem class.

A skeletal plan is constructed in SP-GEN in four phases:

1. In the first phase the execution of the source plan is simulated and explanations for the effects of the individual operations are constructed.

2. In the second phase the generalization of these explanations is performed with respect to a criterion of operationality, that specifies the vocabulary for defining abstract operators for the skeletal plan.

3. In the third phase, a dependency analysis of the resulting operator effects unveils the substantial interactions of the concrete plan.

4. In the fourth phase the concept descriptions for the abstract operators of the skeletal plan are formed by collecting and normalizing the important constraints for each operation that were indicated by the dependencies.

In summary, with the developed knowledge acquisition tools different types of information can thus be intelligently documented: its relevance and sufficiency is assessed, redundancies and contradictions are eliminated and knowledge is thereby verified. 


\section{Domain- and Task-Specific Hypertext as Intelligent Documentation}

The development of an intelligent documentation of the real world knowledge which is relevant for some application such as production planning in mechanical engineering is an important stepping stone for constructing cooperative knowledge based systems.

Since the presented knowledge acquisition method integrates several information sources using different knowledge acquisition tools, and since knowledge is elicited with respect to a model of expertise, there is a need to structure and combine the resulting documents in a sophisticated manner. We propose to build a hypertext with the coordinated knowledge acquisition tools $\mathrm{CECoS}$ and COKAM+ to obtain intelligent documents. Therefore we also may integrate hypertext facilities in the knowledge acquisition method.

In the following we present an example of a possible hypertext, which can be built with CECoS and COKAM+. Further benefits for knowledge-based systems through incorporation of hypertext structures are given in the second part of this chapter.

Figure 6 shows the structure of the hypertext in which data is stored in a network of nodes connected by links. Each case is an instance of a problem class. The problem classes are hierarchically structured, where each class is defined by several features and each feature belongs to a certain category. The hypertext provides access to the classes of the hierarchy, their feature descriptions and all concrete cases which belong to a class. It also includes the visualization of the cases. Through the categorization of the features according to the model of expertise, it is possible to access features with respect to a certain view. For example the feature "long workpiece", which belongs to the 'concrete product view' (a single view), is used to define a terminal class in the problem hierarchy. These proposed hypertext facilities might be of interest in knowledge retrieval under specific aspects.

Analogous to a case each operator, which is part of a production plan, is an instance of an operator class. The hierarchically structured operator classes have dual definitions. The mechanical engineering (ME) descriptions refer to the CNC machine instructions which perform an operation of a certain type. Parallel to this definition, each operator class is also defined in terms of STRIPS-like preconditions and consequences which have been elicited with COKAM+. Abstract and refine relations (also from COKAM+) connect abstract and concrete operator descriptions. The CNC machine instructions, the STRIPS-like operator definitions and the abstract and refine relations, are also categorized according to the identified views.

Obviously the different hierarchical structures and the various task-related links, which make up the domain- and task-specific hypertext allow a better access to the information needed for production planning. The task-specific hypertext links provide an easy access to the information which is relevant for performing the task. For example, if a sequence of machining operations is needed which manufactures a certain type of workpiece, access to an operation sequence or even CNC machine instructions can easily be found via the problem class, into which the manufacturing problem falls. Similarly, an access to segments of CNC instructions may be found through the defined operator classes. The CNC-programs as well as macros are thus explained in terms of a model of expertise and stored accordingly, so that they can be easily accessed for reuse (Redmiles, 1990).

The dependencies which exist among the different information items with respect to the task that is to be performed are explicitly represented by the links of the hypertext, so that the important constraints for achieving the goals of a task are made explicit. What belongs together in a task is also stored closely together.

The hypertext also integrates all the available task-relevant information that was previously separately stored. By cross checking the information from different sources redundancies and contradictions are eliminated and the relevance and sufficiency of the stored information has 


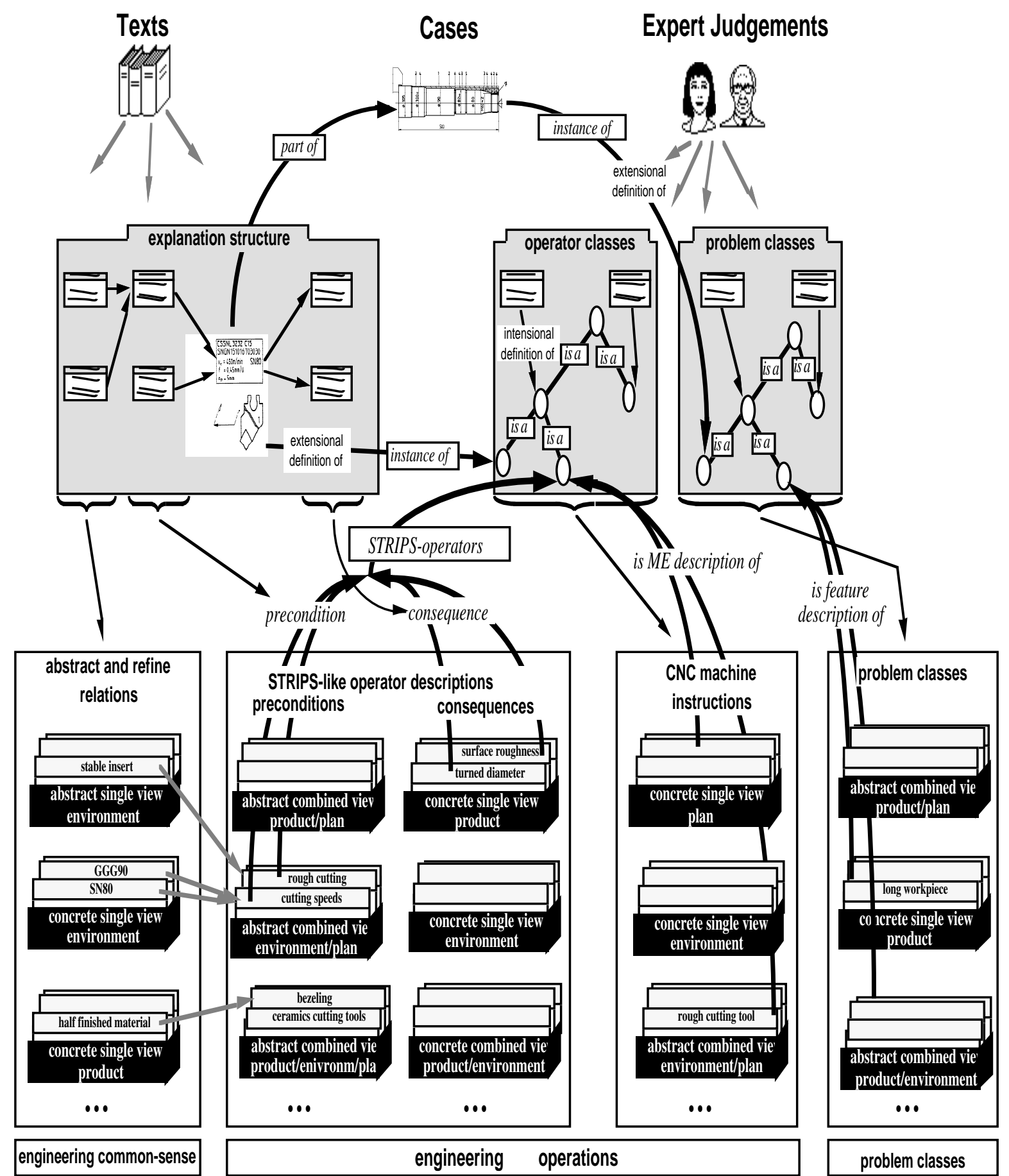

Figure 6: Intelligent documentation of relevant information sources as a domain- and taskspecific hypertext

been assessed. This yields an early knowledge verification. The proposed hypertext facilitates the integration of knowledge from several information sources and different acquisition tools and is thus a more intelligent documentation of the information, available in the field.

Hypertext provides powerful means for intelligently storing the elicitation history of knowledge units. For example, each knowledge unit in the formal knowledge base is linked to its semiformal and informal predecessors. In addition each unit is related to the model of expertise and the domain model, as well as to the respective class. Thus it is easy to follow the formalization process of a knowledge unit and to investigate knowledge units according to a common view or to a common task within production planning. It is even possible to retrieve knowledge units with respect to both of these aspects. Furthermore the context in which a knowledge unit has been elicited can be presented (e.g. the case including the concrete CNC 
program, a graphical presentation, the initial and final states, etc.). So hypertext techniques allow a powerful interface between informal and formal knowledge bases.

The hypertext structure also provides a good facility to generate high level explanations. Thus the user may look up an explanation for each operator generated by the expert system. The operator relies on several preconditions which have been successfully tested by the system, and has some consequences which are suitable to accomplish an intermediate production state. The preconditions and consequences can be explained to the user informally and on different levels of generality through the hypertext structure by following the links from the formal to the informal knowledge.

Since hypertexts provide browsing possibilities, the flexibility for extensions and other advantages, the proposed documentation is already useful in the conventional setting. The human problem solver is supported in finding an appropriate previous case for modification planning, respective CNC-programs and information can be more easily shared between different task categories, thus contributing to the goal of computer-integrated manufacturing.

The hypertext is also structured in accordance with the processes which are performed for constructing a manufacturing plan. It therefore forms an intermediate stage for building a respective knowledge-based system (Schmalhofer, Kühn \& Schmidt, 1991). After the information, which is stored in the different nodes of the hypertext, is transferred into formal representations, the skeletal plan generation procedure SP-GEN can be used to develop a prototypical knowledge base. By striking a balance between the past and future developments, the barriers for innovation can thus be lowered.

\section{Conclusion}

The domain- and task-specific hypertext, which is built with CECoS and COKAM+ yields direct benefits for the human problem solving activities in the current conventional setting. The hypertext is structured according to a model of expertise, which reflects the human problem solving strategies at a general level and which can, in addition, be employed for the top-down design of a knowledge-based system.

Therefore, the proposed intelligent documentation strikes a beneficial balance between the already existing conventional procedures, into which large investments have been made over long time periods and the necessity for introducing innovation via cooperative knowledge-based systems. Since intelligent documentation provides direct benefits and since it is an intermediate state for constructing knowledge based systems, it lowers the barrier for introducing innovation in complex real world domains.

The current conceptual developments of intelligent documentation in form of a domain-specific hypertext were elaborated on the basis of two previous implementations. Although the structures which were constructed by the previous implementations of the knowledge acquisition tools CECoS (Bergmann \& Schmalhofer, 1991), COKAM+ (Schmidt \& Schmalhofer, 1990) and SP-GEN (Schmalhofer, Bergmann, Kühn \& Schmidt, 1991) bear a clear resemblance to the hypertext links shown in Figure 6, the previous versions of CECoS and COKAM+ were independently implemented. The practical experience with the actual implementations supplied essential insights for the development of the new concepts. This paper thus provides the conceptual frame for a coordinated implementation with an extended functionality that yields a domain- and task specific hypertext. 


\section{Acknowledgements}

This research was funded as part of the ARC-TEC project by grant ITW $8902 \mathrm{C} 4$ from BMFT (German ministry for research and technology). We would like to thank Ralph Bergmann, Otto Kühn, Ralf Legleitner and Gabriele Schmidt for their contributions to this work. Discussions on some issues of this paper with Phillip Hanschke, Andreas Lemke, Michel Manago and David Redmiles are much appreciated.

\section{References}

Anderson, J.R. (1990). The adaptive character of thought. Hillsdale, NJ: Lawrence Erlbaum.

Bergmann, R. \& Schmalhofer, F. (1991). CECoS: A case experience combination system for knowledge acquisition for expert systems. Behavior Research Methods, Instruments, \& Computers, 23, 142-148.

Breuker, J. \& Wielinga, B. (1989). Models of expertise in knowledge acquisition. In Guida, G. \& Tasso, C. (Eds.), Topics in expert system design, methodologies and tools (pp. 265 295). Amsterdam, Netherlands: North Holland.

Chase, W.G. \& Ericcson, K.A. (1982). Skill and working memory. In Bower, G.H. (Ed.), The psychology of learning and motivation. New York: Academic Press.

Chi, M., Feltovich, P., \& Glaser, R. (1981). Categorization and representation of physics problems by experts and novices. Cognitive Science, 5, 121-152.

Chien, S.A. (1989). Using and refining simplifications: Explanation-based learning of plans in intractable domains. In Proceedings of the Eleventh International Joint Conference on Artificial Intelligence (pp. 590-595). Palo Alto, CA: Morgan Kaufmann.

Egan, D.E. \& Schwartz, B.J. (1979). Chunking in recall of symbolic drawings. Memory and Cognition, 7, 149-158.

Fischer, G., Lemke, A., \& Mastaglio, T. (1990). Using critics to enpower users. In Chew, J.C. \& Whiteside, J. (Eds.), Human Factors in Computing Systems (CHI '90 Conference Proceedings) (pp. 337-347). Reading, MA: Addison-Wesley.

Friedland, P.E. \& Iwasaki, Y. (1985). The concept and implementation of skeletal plans. Journal of Automated Reasoning, 1, 161-208.

de Groot, A.D. (1966). Perception and memory versus thought: Some old ideas and recent findings. In Kleinmuntz, B. (Ed.), Problem solving. New York: Wiley.

Hammond, K. (1989). Case-based planning. London: Academic Press.

Hoffmann, J. \& Zießler, C. (1983). Objektidentifikation in künstlichen Begriffshierarchien. Zeitschrift für Psychologie, 194, 135-167.

Kühn, O. \& Schmalhofer, F. (1992). Hierarchical skeletal plan refinement: Task and inference structures. In Bauer, C. \& Karbach, W. (Eds.), Proceedings of the 2nd KADS User Meeting (pp. 201-210). München: Siemens AG.

Mitchell, T.M., Keller, R.M., \& Kedar-Cabelli, S.T. (1986). Explanation-based generalization: A unifying view. Machine Learning, 1, 47-80.

Redmiles, D.F. (1990). Explanation to support software reuse. In Proceedings of the AAAI '90 Workshop on Explanation .

Reinartz, T. (1991). Definition von Problemklassen im Maschinenbau als eine Begriffsbildungsaufgabe (DFKI-Document No. D-91-18). Kaiserslautern, Germany: German Research Center for Artificial Intelligence.

Reiser, B.J., Friedmann, P., Gevins, J., Kimberg, D.Y., Ranney, M., \& Romero, A. (1988). A graphical programming language interface for an intelligent Lisp tutor. In Soloway, E., Frye, D., \& Sheppard, S.B. (Eds.), Human Factors in Computing Systems (CHI'88 Conference Proceedings) (pp. 39-44). ACM Press.

Rosch, E. (1978). Principles of categorization. In Rosch, E. \& Lloyd, B. (Eds.), Cognition and categorization. Hillsdale, NJ: Lawrence Erlbaum. 
Schmalhofer, F., Bergmann, R., Kühn, O., \& Schmidt, G. (1991). Using integrated knowledge acquisition to prepare sophisticated expert plans for their re-use in novel situations. In Christaller, T. (Ed.), Proceedings of the 15th German Workshop on Artificial Intelligence (pp. 62-73). Berlin: Springer-Verlag.

Schmalhofer, F., Kühn, O., \& Schmidt, G. (1991). Integrated knowledge acquisition from text, previously solved cases, and expert memories. Applied Artificial Intelligence, 5, 311337.

Schmalhofer, F. \& Thoben, J. (in press). The model-based construction of a case oriented expert system. In Schmalhofer, F., Strube, G., \& Wetter, T. (Eds.), Contemporary knowledge engineering and cognition. Heidelberg: Springer-Verlag.

Schmidt, G. (1992). Knowledge acquisition from text in a complex domain. Paper to be presented at the Fifth International Conference on Industrial \& Engineering Applications of Artificial Intelligence and Expert Systems in Paderborn, Germany.

Schmidt, G., Legleitner, R., \& Schmalhofer, F. (1990). Lautes Denken bei der Erstellung der Schnittaufteilung, der Werkzeugauswahl und Festlegung der Maschineneinstelldaten (Interner Bericht des ARC-TEC Projektes). Kaiserslautern, Germany: German Research Center for Artificial Intelligence.

Schmidt, G. \& Schmalhofer, F. (1990). Case-oriented knowledge acquisition from texts. In Wielinga, B., Boose, J., Gaines, B., Schreiber, G., \& van Someren, M. (Eds.), Current trends in knowledge acquisition (EKAW'90) (pp. 302-312). Amsterdam: IOS Press.

Schmidt, G. \& Wetter, T. (1989). Towards knowledge acquisition in natural language dialogue. In Boose, J.H., Gaines, B.R., \& Ganascia, J.G. (Eds.), Proceedings of the Third European Workshop on Knowledge Acquisition for Knowledge-Based Systems (EKAW '89) (pp. 239-252). Paris, France.

Spur, G. (1979). Produktionstechnik im Wandel. München: Carl Hanser Verlag.

Thoben, J., Schmalhofer, F., \& Reinartz, T. (1991). Wiederholungs-Varianten- und Neuplanung bei der Fertigung rotationssymmetrischer Drehteile (DFKI-Document No. D-91-16). Kaiserslautern, Germany: German Research Center for Artificial Intelligence.

Tschaitschian, B. (1991). Eine integrative Wissenserhebung und-analyse mit CECoS: Konzepte und prototypische Implementierung, Studienarbeit im Fachbereich Informatik, University of Kaiserslautern, Germany.

Wachsmuth, I. \& Meyer-Fujara, J. (1990). Addressing the retrieval problem in large knowledge bases (Reportreihe Modularisierung wissensbasierter Systeme No. 3). Bielefeld, Germany: University of Bielefeld.

Yetim, F. (1991). Eine Hypertext-Komponente zu einem Expertensystem: Benutzerfragen für Erklärungsdialoge. In Maurer, H. (Ed.), Hypertext/Hypermedia '91 (pp. 286-298). Berlin: Springer-Verlag. 


\section{DFKI Publikationen}

Die folgenden DFKI Veröffentlichungen sowie die aktuelle Liste von allen bisher erschienenen Publikationen können von der oben angegebenen Adresse oder per anonymem ftp von ftp.dfki.unikl.de (131.246.241.100) unter pub/Publications bezogen werden.

Die Berichte werden, wenn nicht anders gekennzeichnet, kostenlos abgegeben.

\section{DFKI Research Reports}

RR-92-45

Elisabeth André, Thomas Rist: The Design of Illustrated Documents as a Planning Task 21 pages

\section{RR-92-46}

Elisabeth André, Wolfgang Finkler, Winfried Graf, Thomas Rist, Anne Schauder, Wolfgang Wahlster: WIP: The Automatic Synthesis of Multimodal Presentations 19 pages

\section{RR-92-47}

Frank Bomarius: A Multi-Agent Approach towards Modeling Urban Traffic Scenarios

24 pages

\section{RR-92-48}

Bernhard Nebel, Jana Koehler:

Plan Modifications versus Plan Generation:

A Complexity-Theoretic Perspective

15 pages

\section{RR-92-49}

Christoph Klauck, Ralf Legleitner, Ansgar Bernardi: Heuristic Classification for Automated CAPP 15 pages

RR-92-50

Stephan Busemann:

Generierung natürlicher Sprache

61 Seiten

\section{RR-92-51}

Hans-Jürgen Bürckert, Werner Nutt:

On Abduction and Answer Generation through

Constrained Resolution

20 pages

RR-92-52

Mathias Bauer, Susanne Biundo, Dietmar Dengler, Jana Koehler, Gabriele Paul: PHI - A Logic-Based Tool for Intelligent Help Systems

14 pages
DFKI

-Bibliothek-

PF 2080

67608 Kaiserslautern

FRG

\section{DFKI Publications}

The following DFKI publications or the list of all published papers so far are obtainable from the above address or per anonymous ftp from ftp.dfki.uni-kl.de (131.246.241.100) under pub/Publications.

The reports are distributed free of charge except if otherwise indicated.

\section{RR-92-53}

Werner Stephan, Susanne Biundo:

A New Logical Framework for Deductive Planning 15 pages

RR-92-54

Harold Boley: A Direkt Semantic Characterization of RELFUN

30 pages

\section{RR-92-55}

John Nerbonne, Joachim Laubsch, Abdel Kader Diagne, Stephan Oepen: Natural Language Semantics and Compiler Technology

17 pages

\section{RR-92-56}

Armin Laux: Integrating a Modal Logic of Knowledge into Terminological Logics 34 pages

\section{RR-92-58}

Franz Baader, Bernhard Hollunder:

How to Prefer More Specific Defaults in Terminological Default Logic

31 pages

\section{RR-92-59}

Karl Schlechta and David Makinson: On Principles and Problems of Defeasible Inheritance 13 pages

\section{RR-92-60}

Karl Schlechta: Defaults, Preorder Semantics and Circumscription

19 pages

\section{RR-93-02}

Wolfgang Wahlster, Elisabeth André, Wolfgang Finkler, Hans-Jürgen Profitlich, Thomas Rist: Plan-based Integration of Natural Language and Graphics Generation

50 pages 


\section{RR-93-03}

Franz Baader, Berhard Hollunder, Bernhard Nebel, Hans-Jürgen Profitlich, Enrico Franconi:

An Empirical Analysis of Optimization Techniques for Terminological Representation Systems

28 pages

\section{RR-93-04}

Christoph Klauck, Johannes Schwagereit: GGD: Graph Grammar Developer for features in CAD/CAM

13 pages

RR-93-05

Franz Baader, Klaus Schulz: Combination Techniques and Decision Problems for Disunification 29 pages

\section{RR-93-06}

Hans-Jürgen Bürckert, Bernhard Hollunder, Armin Laux: On Skolemization in Constrained Logics 40 pages

\section{RR-93-07}

Hans-Jürgen Bürckert, Bernhard Hollunder, Armin Laux: Concept Logics with Function Symbols 36 pages

RR-93-08

Harold Boley, Philipp Hanschke, Knut Hinkelmann, Manfred Meyer: COLAB: A Hybrid Knowledge Representation and Compilation Laboratory 64 pages

\section{RR-93-09}

Philipp Hanschke, Jörg Würtz:

Satisfiability of the Smallest Binary Program 8 Seiten

RR-93-10

Martin Buchheit, Francesco M. Donini, Andrea Schaerf: Decidable Reasoning in Terminological Knowledge Representation Systems 35 pages

\section{RR-93-11}

Bernhard Nebel, Hans-Juergen Buerckert: Reasoning about Temporal Relations:

A Maximal Tractable Subclass of Allen's Interval Algebra

28 pages

\section{RR-93-12}

Pierre Sablayrolles: A Two-Level Semantics for French Expressions of Motion 51 pages

\section{RR-93-13}

Franz Baader, Karl Schlechta:

A Semantics for Open Normal Defaults via a Modified Preferential Approach 25 pages

\section{RR-93-14}

Joachim Niehren, Andreas Podelski,Ralf Treinen: Equational and Membership Constraints for Infinite Trees

33 pages

\section{RR-93-15}

Frank Berger, Thomas Fehrle, Kristof Klöckner, Volker Schölles, Markus A. Thies, Wolfgang Wahlster: PLUS - Plan-based User Support Final Project Report

33 pages

\section{RR-93-16}

Gert Smolka, Martin Henz, Jörg Würtz: ObjectOriented Concurrent Constraint Programming in $\mathrm{Oz}$ 17 pages

\section{RR-93-17}

Rolf Backofen:

Regular Path Expressions in Feature Logic

37 pages

\section{RR-93-18}

Klaus Schild: Terminological Cycles and the Propositional $\mu$-Calculus

32 pages

\section{RR-93-20}

Franz Baader, Bernhard Hollunder:

Embedding Defaults into Terminological Knowledge Representation Formalisms

34 pages

\section{RR-93-22}

Manfred Meyer, Jörg Müller:

Weak Looking-Ahead and its Application in Computer-Aided Process Planning 17 pages

\section{RR-93-23}

Andreas Dengel, Ottmar Lutzy:

Comparative Study of Connectionist Simulators 20 pages

\section{RR-93-24}

Rainer Hoch, Andreas Dengel:

Document Highlighting -

Message Classification in Printed Business Letters 17 pages

\section{RR-93-25}

Klaus Fischer, Norbert Kuhn: A DAI Approach to Modeling the Transportation Domain 93 pages

\section{RR-93-26}

Jörg P. Müller, Markus Pischel: The Agent Architecture InteRRaP: Concept and Application 99 pages

\section{RR-93-27}

Hans-Ulrich Krieger:

Derivation Without Lexical Rules

33 pages

RR-93-28

Hans-Ulrich Krieger, John Nerbonne, Hannes Pirker: Feature-Based Allomorphy 8 pages

\section{RR-93-29}

Armin Laux: Representing Belief in Multi-Agent Worlds viaTerminological Logics 35 pages 


\section{RR-93-33}

Bernhard Nebel, Jana Koehler:

Plan Reuse versus Plan Generation: A Theoretical and Empirical Analysis

33 pages

\section{RR-93-34}

Wolfgang Wahlster:

Verbmobil Translation of Face-To-Face Dialogs 10 pages

RR-93-35

Harold Boley, François Bry, Ulrich Geske (Eds.): Neuere Entwicklungen der deklarativen KI-

Programmierung — Proceedings

150 Seiten

Note: This document is available only for a nominal charge of 25 DM (or 15 US-\$).

\section{RR-93-36}

Michael M. Richter, Bernd Bachmann, Ansgar Bernardi, Christoph Klauck, Ralf Legleitner,

Gabriele Schmidt: Von IDA bis IMCOD:

Expertensysteme im CIM-Umfeld

13 Seiten

\section{RR-93-38}

Stephan Baumann: Document Recognition of Printed Scores and Transformation into MIDI 24 pages

\section{RR-93-40}

Francesco M. Donini, Maurizio Lenzerini, Daniele Nardi, Werner Nutt, Andrea Schaerf:

Queries, Rules and Definitions as Epistemic Statements in Concept Languages

23 pages

\section{RR-93-41}

Winfried H. Graf: LAYLAB: A Constraint-Based Layout Manager for Multimedia Presentations 9 pages

\section{RR-93-42}

Hubert Comon, Ralf Treinen:

The First-Order Theory of Lexicographic Path Orderings is Undecidable

9 pages

RR-93-44

Martin Buchheit, Manfred A. Jeusfeld, Werner Nutt, Martin Staudt: Subsumption between Queries to Object-Oriented Databases 36 pages

\section{RR-93-45}

Rainer Hoch: On Virtual Partitioning of Large Dictionaries for Contextual Post-Processing to Improve Character Recognition 21 pages

\section{RR-93-46}

Philipp Hanschke: A Declarative Integration of Terminological, Constraint-based, Data-driven, and Goal-directed Reasoning

81 pages

\section{DFKI Technical Memos}

\section{TM-91-15}

Stefan Busemann: Prototypical Concept Formation An Alternative Approach to Knowledge Representation 28 pages

\section{TM-92-01}

Lijuan Zhang: Entwurf und Implementierung eines Compilers zur Transformation von

Werkstückrepräsentationen

34 Seiten

TM-92-02

Achim Schupeta: Organizing Communication and Introspection in a Multi-Agent Blocksworld 32 pages

TM-92-03

Mona Singh:

A Cognitiv Analysis of Event Structure

21 pages

\section{TM-92-04}

Jürgen Müller, Jörg Müller, Markus Pischel, Ralf Scheidhauer:

On the Representation of Temporal Knowledge 61 pages

\section{TM-92-05}

Franz Schmalhofer, Christoph Globig, Jörg Thoben: The refitting of plans by a human expert 10 pages

\section{TM-92-06}

Otto Kühn, Franz Schmalhofer: Hierarchical skeletal plan refinement: Task- and inference structures 14 pages

\section{TM-92-08}

Anne Kilger: Realization of Tree Adjoining Grammars with Unification

27 pages

\section{TM-93-01}

Otto Kühn, Andreas Birk: Reconstructive Integrated Explanation of Lathe Production Plans 20 pages

\section{TM-93-02}

Pierre Sablayrolles, Achim Schupeta:

Conlfict Resolving Negotiation for COoperative Schedule Management

21 pages

\section{TM-93-03}

Harold Boley, Ulrich Buhrmann, Christof Kremer: Konzeption einer deklarativen Wissensbasis über recyclingrelevante Materialien 11 pages

\section{TM-93-04}

Hans-Günther Hein: Propagation Techniques in WAM-based Architectures — The FIDO-III Approach 105 pages 


\section{DFKI Documents}

D-92-23

Michael Herfert: Parsen und Generieren der Prolog artigen Syntax von RELFUN

51 Seiten

\section{D-92-24}

Jürgen Müller, Donald Steiner (Hrsg.):

Kooperierende Agenten

78 Seiten

D-92-25

Martin Buchheit: Klassische Kommunikations- und Koordinationsmodelle

31 Seiten

\section{D-92-26}

Enno Tolzmann:

Realisierung eines Werkzeugauswahlmoduls mit

Hilfe des Constraint-Systems CONTAX

28 Seiten

\section{D-92-27}

Martin Harm, Knut Hinkelmann, Thomas Labisch: Integrating Top-down and Bottom-up Reasoning in COLAB

40 pages

\section{D-92-28}

Klaus-Peter Gores, Rainer Bleisinger: Ein Modell zur Repräsentation von Nachrichtentypen

56 Seiten

\section{D-93-01}

Philipp Hanschke, Thom Frühwirth: Terminological Reasoning with Constraint Handling Rules 12 pages

\section{D-93-02}

Gabriele Schmidt, Frank Peters,

Gernod Laufkötter: User Manual of COKAM+ 23 pages

\section{D-93-03}

Stephan Busemann, Karin Harbusch(Eds.):

DFKI Workshop on Natural Language Systems:

Reusability and Modularity - Proceedings

74 pages

\section{D-93-04}

DFKI Wissenschaftlich-Technischer Jahresbericht 1992

194 Seiten

\section{D-93-05}

Elisabeth André, Winfried Graf, Jochen Heinsohn, Bernhard Nebel, Hans-Jürgen Profitlich, Thomas Rist, Wolfgang Wahlster:

PPP: Personalized Plan-Based Presenter

70 pages

\section{D-93-06}

Jürgen Müller (Hrsg.):

Beiträge zum Gründungsworkshop der Fachgruppe

Verteilte Künstliche Intelligenz Saarbrücken 29.-30. April 1993

235 Seiten

Note: This document is available only for a nominal charge of 25 DM (or 15 US-\$).

\section{D-93-07}

Klaus-Peter Gores, Rainer Bleisinger:

Ein erwartungsgesteuerter Koordinator zur partiellen

Textanalyse

53 Seiten

\section{D-93-08}

Thomas Kieninger, Rainer Hoch: Ein Generator mit Anfragesystem für strukturierte Wörterbücher zur Unterstützung von Texterkennung und Textanalyse 125 Seiten

\section{D-93-09}

Hans-Ulrich Krieger, Ulrich Schäfer:

TDL ExtraLight User's Guide

35 pages

D-93-10

Elizabeth Hinkelman, Markus Vonerden,Christoph Jung: Natural Language Software Registry

(Second Edition)

174 pages

D-93-11

Knut Hinkelmann, Armin Laux (Eds.):

DFKI Workshop on Knowledge Representation

Techniques - Proceedings

88 pages

D-93-12

Harold Boley, Klaus Elsbernd, Michael Herfert, Michael Sintek, Werner Stein:

RELFUN Guide: Programming with Relations and Functions Made Easy

86 pages

D-93-14

Manfred Meyer (Ed.): Constraint Processing Proceedings of the International Workshop at CSAM'93, July 20-21, 1993

264 pages

Note: This document is available only for a nominal charge of $25 \mathrm{DM}$ (or 15 US-\$).

D-93-15

Robert Laux: Untersuchung maschineller

Lernverfahren und heuristischer Methoden im Hinblick auf deren Kombination zur Unterstützung eines Chart-Parsers

86 Seiten

\section{D-93-20}

Bernhard Herbig:

Eine homogene Implementierungsebene für einen hybriden Wissensrepräsentationsformalismus 97 Seiten

\section{D-93-21}

Dennis Drollinger:

Intelligentes Backtracking in Inferenzsystemen am Beispiel Terminologischer Logiken 53 Seiten 